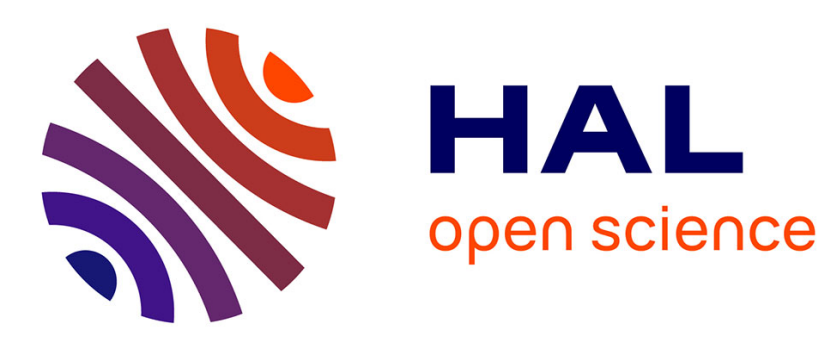

\title{
A Fibrational Account of Local States
}

Kenji Maillard, Paul-André Melliès

\section{To cite this version:}

Kenji Maillard, Paul-André Melliès. A Fibrational Account of Local States. 2015 30th Annual ACM/IEEE Symposium on Logic in Computer Science (LICS), Jul 2015, Kyoto, France. pp.402413, 10.1109/LICS.2015.45 . hal-03066087

\section{HAL Id: hal-03066087 https://hal.inria.fr/hal-03066087}

Submitted on 15 Dec 2020

HAL is a multi-disciplinary open access archive for the deposit and dissemination of scientific research documents, whether they are published or not. The documents may come from teaching and research institutions in France or abroad, or from public or private research centers.
L'archive ouverte pluridisciplinaire HAL, est destinée au dépôt et à la diffusion de documents scientifiques de niveau recherche, publiés ou non, émanant des établissements d'enseignement et de recherche français ou étrangers, des laboratoires publics ou privés. 


\section{A Fibrational Account of Local States}

\author{
Kenji Maillard \\ ENS Ulm \\ Paris, France
}

\author{
Paul-André Melliès \\ Laboratoire Preuves, Programmes, Systèmes \\ CNRS, Université Paris Diderot \\ Paris, France
}

\begin{abstract}
One main challenge of the theory of computational effects is to understand how to combine various notions of effects in a meaningful way. Here, we study the particular case of the local state monad, which we would like to express as the result of combining together a family of global state monads parametrized by the number of available registers. To that purpose, we develop a notion of indexed monad inspired by the early work by Street, which refines and generalizes Power's recent notion of indexed Lawvere theory. One main achievement of the paper is to integrate the block structure necessary to encode allocation as part of the resulting notion of indexed state monad. We then explain how to recover the local state monad from the functorial data provided by our notion of indexed state monad. This reconstruction is based on the guiding idea that an algebra of the indexed state monad should be defined as a section of a 2-categorical notion of fibration associated to the indexed state monad by a Grothendieck construction.

Index Terms-Computational effects; algebraic theories; Lawvere theories; state monad; local state monad; 2-categories; fibration
\end{abstract}

\section{INTRODUCTION}

Despite years of intensive mathematical study, the semantic nature of memory and state in programming languages has not yet revealed all its secrets, and thus remains particularly interesting and important to investigate today - in particular because it combines and interleaves two entirely different aspects :

- on the one hand, each memory register can be read and written using dedicated lookup and update operations available as effects to the programmer,

- on the other hand, memory registers can be allocated and deallocated at any time, depending on the memory management policy, as well as on the needs and desires of the very same programmer.

These two complementary aspects of memory have been thoroughly studied in the semantic literature. The primary focus on allocation and deallocation mechanisms in higherorder imperative languages like Algol have lead pionneer researchers like Oles and Reynolds to promote the idea that types should be interpreted as presheaves over combinatorial categories describing the memory shapes and resources [17], [23]. This simple idea had an impressive posterity in our field, including separation logic [24], [22] and nominal sets [18]. A traditional example of such a combinatorial category of memory shapes, also related to separation logic and nominal

This work has been partly supported by the ANR RECRE project sets, is provided by the category Inj with natural numbers as objects, seen as finite cardinals $[n]=\{0, \ldots, n-1\}$, and injections between them.

In parallel, and in a somewhat independent strand of research, Moggi realized that it was possible to understand the read and write operations of a memory register as a specific monadic effect living on top of a purely functional language [15]. This monadic account of states works as follows. Suppose that $n$ denotes the number of registers allocated in your computer, and that $S$ denotes the set of states possibly assigned to any of these $n$ registers. In this case, one defines a monad (called the state monad)

$$
T_{n} \quad: \quad \text { Set } \longrightarrow \text { Set }
$$

on the category Set of sets and functions, by :

$$
T_{n} \quad: \quad A \quad \mapsto \quad S^{n} \Rightarrow\left(S^{n} \times A\right)
$$

where each element of $S^{n}$ is a finite list

$$
\left(s_{0}, \ldots, s_{n-1}\right) \quad \in S^{n}
$$

consisting of the states of each allocated register. A typical instance of such a set of states (or values) is provided by $S=V=\{$ true, false $\}$ where each register is thus meant to contain a bit. On some occasions, we find useful to call mnemoid (for set with memory) or more precisely $n$-mnemoid an algebra $A$ of the state monad $T_{n}$.

\section{a) The local state monad}

For many years, these two semantic approaches to memory and states in higher-order programming language remained largely disconnected. The situation drastically changed when Plotkin and Power, inspired by discussions with O'Hearn, exhibited a monad (called the local state monad)

$$
T \quad: \quad[\text { Inj, Set }] \quad \longrightarrow \quad[\text { Inj,Set }]
$$

on the category $[I n j, S e t]$ of covariant presheaves over the category Inj of finite sets and injections. This monad is defined on a given presheaf $A$ by the slightly intimidating co-end formula

$$
T A: n \mapsto S^{n} \Rightarrow\left(\int^{p \in I n j} S^{p} \times A_{p} \times \operatorname{Inj}(n, p)\right)
$$

whose purpose is to adapt the definition of the traditional state monad $T_{n}$ on Set to the presheaf category $[\operatorname{Inj}$, Set]. In their 
seminal paper, Plotkin and Power established in particular that the local state monad $T$ just defined implements at the same time the lookup, update, and allocation operations expected of a state monad on the presheaf category $[$ Inj, Set $]$. This result is technically established by formulating a purely algebraic description of the algebras $A$ of the local state monad $T$. This paper by Plotkin and Power together with later elaborations [25], [12] characterize such an algebra $A$ as a family of sets $\left(A_{n}\right)_{n \in \mathbb{N}}$ indexed by natural numbers

$$
\begin{array}{llllll}
A_{0} & A_{1} & A_{2} & A_{3} & A_{4} & \ldots
\end{array}
$$

equipped with five families of operations, described as follows in the case when $S=\{$ true, false $\}$. First of all, for each natural number $n \in \mathbb{N}$ and each location $l o c \in[n]$, there is a binary lookup operation

$$
\text { lookup }_{\langle l o c\rangle}: A_{n} \times A_{n} \longrightarrow A_{n}
$$

which intuitively behaves like a conditional test, and branches on its left operand when the value of the register $l o c$ is true, and on its right operand when the value of the register loc is false. There is also an update operation for each value val $\in$ $\{$ true, false $\}$ possibly assigned to the register $l o c \in[n]$ :

$$
\text { update }_{\langle l o c, v a l\rangle}: A_{n} \longrightarrow A_{n}
$$

Then, for each natural number $n \in \mathbb{N}$, for each location $l o c \in$ $[n+1]$ and for each value val $\in\{$ true, false $\}$, there is an operation

$$
\operatorname{fresh}_{\langle l o c, v a l\rangle}: A_{n+1} \quad \longrightarrow \quad A_{n}
$$

whose intuitive purpose is to allocate among $n$ registers a fresh register at location $l o c \in[n+1]$ moreover initialized with the value val $\in\{$ true, false $\}$. Then, for each natural number $n \in \mathbb{N}$ and for each location $l o c \in[n+1]$, there is an operation

$$
\operatorname{collect}_{\langle l o c\rangle}: A_{n} \quad \longrightarrow \quad A_{n+1}
$$

whose intuitive purpose is to deallocate or garbage collect the register at location $l o c \in[n+1]$. Finally, for each natural number $n \in \mathbb{N}$ and for each pair of locations $l o c, l o c+1 \in[n]$, there is an operation

$$
\text { permute }_{\langle l o c, l o c+1\rangle}: A_{n} \quad \longrightarrow \quad A_{n}
$$

whose intuitive purpose is to permute the two registers at location $l o c$ and $l o c+1$. These five families of operations are moreover regulated by a natural but also pretty long series of equations carefully enumerated and depicted as string diagrams in [12].

\section{b) The three groups of operations}

Once this algebraic presentation of the local state monad $T$ has been achieved, a natural question is to understand what it can teach us about the very construction and nature of the local state monad $T$ itself. A preliminary observation is that the five families of operations can be organized in three groups :

1) for a given natural number $n$, the operations lookup and update of degree $n$ provide the set $A_{n}$ with the structure of $n$-mnemoid - that is, of an algebra of the state monad $T_{n}$ on $n$ registers introduced earlier,

2) the operations collect and permute provide together the presheaf structure of the family of sets $\left(A_{n}\right)_{n \in \mathbb{N}}$ on the category $I n j$ of finite sets and injections,

3) the algebraic purpose of the allocation operation fresh remains something of a mystery at this stage - this is a serious conceptual concern, since allocation plays a central role in the definition of the local state monad $T$, and we thus wish to resolve it in the present paper.

The algebraic presentations by Staton [25] and Melliès [12] of the local state monad were to a large extent designed to clarify how these three groups of operations and equations are intertwined in the local state monad. Here, we would like to revisit this analysis starting from a slightly different angle, offered by the elegant and compelling fibrational point of view recently advocated and developed by Power [20].

\section{c) Indexed Lawvere theories}

In his work, Power observes that the groups of operations (1) and (2) may be combined by defining a functor

$$
\mathcal{T} \quad: \quad \text { Inj } \longrightarrow \text { Law }
$$

from the category $I n j$ to the category Law of Lawvere theories and finite product preserving functors between them. Such a functor $\mathcal{T}$ is called by Power an indexed Lawvere theory. The functor $\mathcal{T}$ transports every natural number $n$ to the algebraic theory $\mathcal{T}_{n}$ associated to the state $\operatorname{monad} T_{n}$, which is indeed finitary. This construction relies on the fact that every injection $f: m \rightarrow n$ induces a morphism

$$
\mathcal{T}_{f}: \mathcal{T}_{m} \quad \longrightarrow \mathcal{T}_{n}
$$

defined by relabelling along $f$ the register locations $l o c \in[m]$ used by the operations

$$
\text { lookup }_{\langle l o c\rangle} \quad \text { update }_{\langle l o c, v a l\rangle}
$$

of degree $m$ into the corresponding operations

$$
\text { lookup }_{\langle f(l o c)\rangle} \quad \text { update }_{\langle f(l o c), v a l\rangle}
$$

of degree $n$. This establishes that the read and write operations of the local state monad define something which deserves the name of presheaf of operations.

\section{d) Models of Indexed Lawvere theories}

The indexed Lawvere theory $\mathcal{T}$ induces a notion of model defined by Power as a family of models (in the usual sense)

$$
\begin{array}{llllll}
A_{0} & A_{1} & A_{2} & A_{3} & A_{4} & \ldots
\end{array}
$$

of the Lawvere theory $\mathcal{T}_{n}$ of the corresponding degree $n$. Note that each such model $A_{n}$ may be alternatively seen as a $n$-mnemoid, that is, as an algebra of the state monad $T_{n}$. This family of models is moreover required to satisfy a series of coherence conditions recalled in Section II. Although he does not state it exactly in that way, an important observation by Power is that a model $\left(A_{n}\right)_{n \in \mathbb{N}}$ of the indexed Lawvere theory $\mathcal{T}$ in his sense is the same thing as a family of 
sets $\left(A_{n}\right)_{n \in \mathbb{N}}$ equipped with the operations and equations of the two groups (1) and (2) of the local state monad $T$. This result establishes that the indexed Lawvere theory $\mathcal{T}$ provides a precise description of the local state monad $T$ as a combination of the state monads $T_{n}$, as long as this monad $T$ is restricted to the two groups (1) and (2) of operations and equations. As such, the indexed Lawvere theory $\mathcal{T}$ is not able to capture the allocation mechanisms at work in the local state monad $T$.

\section{e) Block structure for allocation}

In order to fill the gap with the local state monad $T$, Power introduces what he calls a block structure on the models of his indexed Lawvere theory $\mathcal{T}$. A block structure as defined in [20] is a family of homomorphisms

$$
\text { block }_{n}: A_{n+1} \longrightarrow V \Rightarrow A_{n}
$$

between models of the Lawvere theory $\mathcal{T}_{n+1}$. The very definition of block structure relies on the key observation that the exponentiation

$$
V \Rightarrow-\quad: \quad A \quad \mapsto \quad V \Rightarrow A
$$

by the set $V$ of values possibly assigned to a register, defines a functor between the categories of models

$$
V \Rightarrow-\quad: \quad \mathcal{T}_{n}-\operatorname{Mod} \quad \longrightarrow \quad \mathcal{T}_{n+1}-\operatorname{Mod}
$$

of the Lawvere theories $\mathcal{T}_{n}$ and $\mathcal{T}_{n+1}$. Another equivalent way to look at it, is to view the exponentiation by $V$ as a functor

$$
V \Rightarrow-\quad: \quad T_{n}-A l g \quad \longrightarrow \quad T_{n+1}-A l g
$$

between the categories of $n$-mnemoids (or $T_{n}$-algebras) and $(n+1)$-mnemoids (or $T_{n+1}$-algebras). This family of homomorphisms block $n$ between $(n+1)$-mnemoids is moreover required to make two coherence diagrams commute, recalled in Section II. Thanks to this notion of block structure, Power establishes the following striking result :

Theorem (Power 2011) The category of algebras of the local state monad $T$ is equivalent to the category of models of the indexed Lawvere theory $\mathcal{T}$ equipped with a block structure.

This result is important conceptually because it pinpoints the exact algebraic structure missing in order to fill the gap between the indexed Lawvere theory $\mathcal{T}$ which does not handle allocation and the local state monad $T$ which does. From that point of view, the notion of block structure appears as some kind of algebraic "glue" necessary (and at the same time sufficient) in order to combine the various state monads $T_{n}$ and to obtain the local state monad $T$ in the end. However, Power himself recognizes in his paper that the notion of block structure remains somewhat unsatisfactory in the way it is formulated, and that it thus deserves to be further elaborated. This is precisely what we intend to do in the present paper, by investigating the algebraic and conceptual nature of the notion of block structure, using 2-categorical ideas.

\section{f) Indexed monads}

A good starting point for our journey is to think of an "indexed Lawvere theory" as an algebraic refinement of the traditional notion of "indexed category". Recall that a $\mathscr{C}$ indexed category is defined as a (contravariant) pseudo-functor

$$
F \quad: \mathscr{C}^{o p} \longrightarrow C a t
$$

from a basis category $\mathscr{C}$ to the category $C$ at of small categories. There is a well-known correspondence between such $\mathscr{C}$-indexed categories and cloven fibrations over the category $\mathscr{C}$. This correspondence relies on the Grothendieck construction which associates to every $\mathscr{C}$-indexed category a cloven fibration

$$
\pi_{F}: \int F \quad \longrightarrow \mathscr{C}
$$

whose fiber $\pi_{F}^{-1}(c)$ over an object $c$ of the category $\mathscr{C}$ coincides with the category $F(c)$. The ongoing discussion on the local state monad $T$ and the indexed Lawvere theory $\mathcal{T}$ leads us to introduce the notion of indexed monad, defined as a (contravariant) pseudo-functor

$$
\mathscr{T}: \mathscr{C}^{o p} \longrightarrow \text { Mnd }
$$

from the base category $\mathscr{C}$ to the 2-category $M n d$ introduced by Street [10] in his celebrated paper on the formal theory of monads. Recall that the objects of the 2-category $M n d$ are pairs $(\mathscr{E}, T)$ consisting of a category $\mathscr{E}$ together with a monad on it :

$$
T: \mathscr{E} \longrightarrow \mathscr{E} .
$$

As expected, the usual 2-category $C a t$ of categories, functors and natural transformations defines a sub-2-category of $M n d$ where a category $\mathscr{E}$ living in $C a t$ is seen in $M n d$ as the pair $(\mathscr{E}, I d)$ consisting of the category $\mathscr{E}$ equipped with the identity monad $I d$. An indexed category $F$ is thus a specific case of indexed monad $\mathscr{T}$. At the same time, there exists a functor

$$
\iota \quad: \quad \text { Law }^{o p} \longrightarrow M n d
$$

which transports every Lawvere theory $\mathcal{T}$ to the pair $($ Set,$T)$ consisting of the category Set together with the finitary monad $T$ associated to the Lawvere theory $\mathcal{T}$. The contravariant nature of this functor $\iota$ has to do with Street's very definition of the 2-category $M n d$. From that follows that every indexed Lawvere theory

$$
\mathcal{T}: \mathscr{C} \quad \longrightarrow \quad \text { Law }
$$

in the sense of Power gives rise to a $\mathscr{C}$-indexed monad

$$
\mathscr{T}: \mathscr{C}^{o p} \longrightarrow M n d
$$

obtained by composition with $\iota$. 


\section{g) Block structures integrated as part of indexed monads}

The key observation of the present paper is that the block structure introduced by Power may be smoothly integrated in the conceptual framework offered by indexed monads. This is achieved along the following idea. In our alternative account, the indexed Lawvere theory $\mathcal{T}$ formulated by Power is replaced by an indexed monad

$$
\mathscr{T} \quad: \quad \operatorname{Inj}^{o p} \longrightarrow \text { Mnd }
$$

which transports every natural number $n$ to the pair $\left(\operatorname{Set}, T_{n}\right)$ consisting of the category $S e t$ and the state monad $T_{n}$. Similarly, every injection $f: m \rightarrow n$ is transported into a morphism

$$
\mathscr{T}(f) \quad: \quad\left(\operatorname{Set}, T_{n}\right) \quad \longrightarrow \quad\left(\operatorname{Set}, T_{m}\right)
$$

of the 2-category $M n d$. Note in particular the change of orientation, reflecting the contravariant definition of indexed monads. It is worth observing that this morphism (2) in $M n d$ is entirely characterized by a monad morphism

$$
T_{f}: T_{m} \quad \longrightarrow \quad T_{n}
$$

which performs the same relabelling along $f$ as explained previously in (1). So, the key novelty with respect to indexed Lawvere theories is that the notion of morphism in the category $M n d$ is in fact more expressive than the notion of morphism in the category Law. In particular, it is sufficiently expressive to incorporate the functor

$$
V \Rightarrow-\quad: \quad T_{n}-A l g \quad \longrightarrow \quad T_{n+1}-A l g
$$

as part of a morphism

$$
\text { Block : }\left(\operatorname{Set}, T_{n}\right) \quad \longrightarrow \quad\left(\operatorname{Set}, T_{n+1}\right)
$$

of the 2-category Mnd. This observation leads us to the idea of extending the category $I n j$ with a family of morphisms

$$
\text { alloc }:[n+1] \longrightarrow[n]
$$

which would be transported by the indexed monad to the Mnd-morphism Block

$$
\mathscr{T}(\text { alloc })=\text { Block } \quad: \quad\left(\operatorname{Set}, T_{n}\right) \quad \longrightarrow \quad\left(\text { Set }, T_{n+1}\right)
$$

in the same way as the injections $f$ are transported by $\mathscr{T}$ to the $M n d$-morphism $T_{f}$. Guided by this intuition, we construct an indexed monad

$$
\mathscr{T} \quad: \text { PInj }^{o p} \longrightarrow \text { Mnd }
$$

which extends the indexed monad $\mathscr{T}$ by shifting from the category $I n j$ to the 2-category $\mathcal{P} I n j$ of partial injections as described in Section IV where, as we have just explained, the canonical morphism

$$
\text { alloc }:[1] \quad \longrightarrow \quad[0]
$$

in the category $\mathcal{P} I n j$ is transported to the morphism Block in the 2-category Mnd.

\section{h) Main theorem of the paper}

The main theorem of our paper relies on the extension and adaptation to indexed monads of the usual Grothendieck construction for indexed categories. The resulting Grothendieck construction associates to every $\mathscr{C}$-indexed monad

$$
\mathscr{T} \quad: \quad c \mapsto\left(\mathscr{E}_{c}, T_{c}\right): \mathscr{C}^{o p} \longrightarrow \text { Mnd }
$$

a 2-categorical notion of fibration (or 2-fibration) in the sense of Hermida, whose fiber above a given object $c$ coincides with the category $T_{c}-A l g$ of algebras associated to the monad $T_{c}$ over the category $\mathscr{E}_{C}$. We then define an algebra of such an indexed monad $\mathscr{T}$ as a section of this 2-fibration. In the case of the indexed state monad, one recovers in this way a family of sets

$$
\begin{array}{llllll}
A_{0} & A_{1} & A_{2} & A_{3} & A_{4} & \ldots
\end{array}
$$

where each $A_{n}$ is an $n$-mnemoid, that is, an algebra of the state monad $T_{n}$ on the category $\mathscr{E}_{n}=\operatorname{Set}$. Our main technical contribution in this paper is to establish that the coherence conditions required on this family of mnemoids in order to define an algebra of the local state monad $T$ in the category $[I n j, S e t]$, are in fact entirely handled by the 2 -fibrational structure associated to a specific indexed state monad $\mathscr{T}$, fully described in the paper.

Theorem There is a correspondence between the algebras of the local state monad $T$ and the sections of the 2-fibration

$$
\pi_{\mathscr{T}} \quad: \quad \int \mathscr{T} \quad \longrightarrow \mathcal{P} \text { Inj. }
$$

This correspondence defines moreover an equivalence of categories between the two concepts.

The description of an algebra $A$ of the local state monad $T$ as a section of the 2-fibration $\pi_{\mathscr{T}}$ may be reformulated in an even simpler way, by observing that such a section is the same thing as a 2-dimensional cell

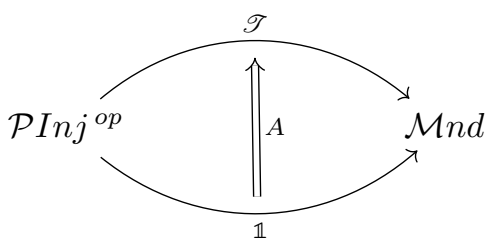

in the 2-category of 2-categories, 2-functors, and colax natural transformations. Here, $\mathbb{1}$ denotes the constant 2 -functor which transports every natural number $n$ to the terminal category. Since $\mathbb{1}$ is also terminal in the category $\left[\mathcal{P} I n j^{o p}, M n d\right]$ of colax natural transformations, this diagram enables one to see an algebra $A$ of the indexed monad $\mathscr{T}$ as a global element of the indexed monad itself.

Despite the need for climbing one degree of abstraction, and working with a 2-categorical notion of fibration, we believe that this reconstruction of the local state monad is sufficiently general and conceptual to shed light on its true nature. A number of new ideas and techniques emerged in the course of the construction, which we did our best to describe as 
meticulously as possible in the course of the paper. The paper is organized in five sections. Section II is devoted to an analysis of the block structure introduced by Power. This analysis leads us to introduce a 2-categorical Grothendieck construction on monads in Section III. Then in Section IV we explain how to apply the 2-categorical framework just defined to the particular case of the local state monad $T$ and establish our main theorem. Finally, we relate in Section V our construction to the algebraic presentation of the local state monad $T$ formulated by Melliès [12], before concluding the paper in Section VI.

\section{BLOCKS IN POWER'S WORK}

In this section, we revisit Power's work and show how it leads us to investigate indexed monads. We first present in detail Power's notion of model of an indexed Lawvere theory. This is then applied to the particular case of the indexed Lawvere theory $\mathcal{T}$ for local state. As explained in the introduction, Power needed to introduce a notion of blockstructure algebra on these models in order to recover the traditional local state monad. A meticulous investigation of this block algebra structure exhibits its fibrational nature.

\section{a) Model of an indexed Lawvere theory}

A model of an indexed Lawvere theory

$$
F: \mathscr{C} \quad \longrightarrow \quad L a w
$$

is given by a family $\left(M_{c}\right)_{c \in \mathscr{C}}$ of models where $M_{c}$ is a model of $F(c)$. Moreover, this assignment must be functorial, meaning that for any 1-cell $f: c \rightarrow c^{\prime}$ in $\mathscr{C}$ there is a natural transformation $M_{f}$ filling the following diagram

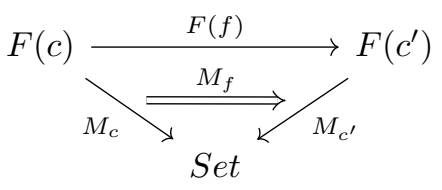

and such that

$$
M_{i d}=i d \quad M_{f \circ g}=M_{f} \cdot M_{g}
$$

A more classical presentation of this notion can be given by considering the functor

$$
\mathscr{C}^{o p} \stackrel{F^{o p}}{\longrightarrow} L_{a w}{ }^{o p} \stackrel{\text { Mod }}{\longrightarrow} \mathcal{C} a t
$$

where Mod: Law $\rightarrow \mathcal{C}$ at is the functor sending a Lawvere theory to its category of models. Note then that the above diagrams and equations is the same thing as what is sometimes called a colax cone over this functor with apex the terminal category $*$ :

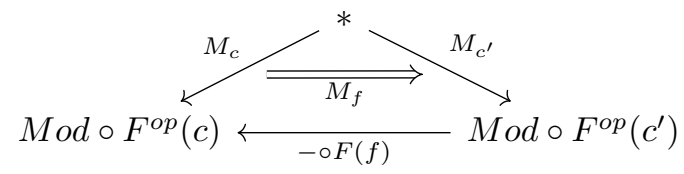

That means that we have an equivalence of categories between the category of models of the indexed Lawvere theory $F$ and the category of colax transformations from the terminal functor $\mathbb{1}: \mathscr{C}^{o p} \rightarrow \mathcal{C}$ at to $F$

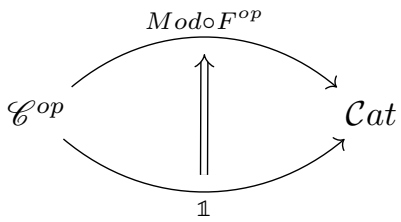

As such, the models of the indexed Lawvere theory $F$ coincides with sections of the fibration

$$
\pi \quad: \quad \operatorname{Mod} \circ F^{o p} \longrightarrow \mathscr{C}
$$

obtained through the Grothendieck construction. Indeed, a section $s$ of $\pi$ is a functor

$$
s: \mathscr{C} \longrightarrow \int \operatorname{Mod} \circ F^{o p}
$$

such that $\pi \circ s=i d_{\mathscr{C}}$. Such a section sends

- an object $c \in \mathscr{C}$ to an element $s(c)=M_{c} \in \operatorname{Mod} \circ$ $F^{o p}(c)$, that is a model of $F(c)$,

- a 1-cell $f: c \rightarrow c^{\prime}$ to a natural transformation

$$
s(f)=M_{f} \quad: \quad M_{c} \quad \longrightarrow \quad F(f)^{*}\left(M_{c^{\prime}}\right)=M_{c^{\prime}} \circ F(f)
$$

\section{b) The block structure}

Let us focus on the case of the indexed Lawvere theory of local state $\mathcal{T}$. A model $A$ of this theory is a family

$$
\begin{array}{llllll}
A_{0} & A_{1} & A_{2} & A_{3} & A_{4} & \ldots
\end{array}
$$

where $A_{i}$ is a model of the Lawvere theory $\mathcal{T}_{n}$ of state on $n$ registers. Moreover, for each injection $f:[m] \rightarrow[n]$, there is a morphism of $\mathcal{T}_{m}$-models

$$
A_{f} \quad: \quad A_{m} \quad \longrightarrow \quad A_{n} \circ \mathcal{T}_{f}
$$

With this data, we can interpret :

- the operations lookup and update in each $A_{n}$,

- the operations collect and permute as injections acting through the coercions between the fibers.

For instance, the injection $i_{n} \in \operatorname{Inj}([n],[n+1])$ sending each natural number to itself allow to interpret the operation

$$
\operatorname{collect}_{\langle n\rangle}=A_{i_{n}} \quad: \quad A_{n} \quad \longrightarrow \quad A_{n+1}
$$

and the bijection $s_{n}^{n+1} \in \operatorname{Inj}([n+2],[n+2])$ only swapping $n$ and $n+1$ enable to interpret the operation

$$
\text { permute }_{\langle n, n+1\rangle}=A_{s_{n}^{n+1}} \quad: \quad A_{n+2} \quad \longrightarrow \quad A_{n+2}
$$

Now, in order to obtain all the operations present in the local state monad, we are left with interpreting the family of operations fresh. For this purpose, Power introduced the notion of a block structure on a model $A$ of the indexed Lawvere theory for local state $\mathcal{T}$. This model $A$ is a block algebra when it is equipped with a family

$$
\operatorname{block}_{\langle n\rangle}: A_{n+1} \quad \longrightarrow \quad V \Rightarrow A_{n}
$$


of $\mathcal{T}_{n+1}$-homomorphims which interact soundly with the two families of operations collect and permute, that is such that the two following diagrams commute :
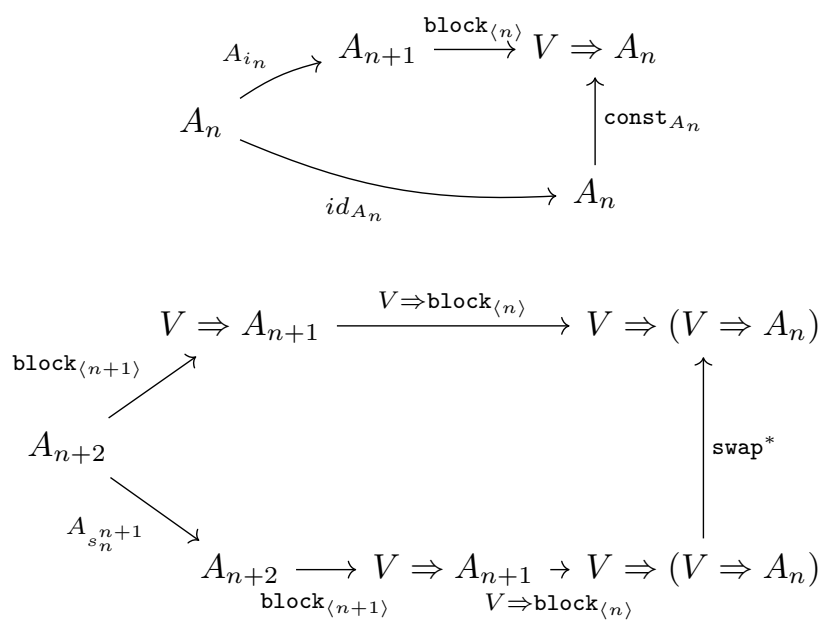

(5)

Here, the arrow const $A_{n}: A_{n} \rightarrow V \Rightarrow A_{n}$ is the natural transformation sending an element $x$ to the constant function with value $x$ and swap* is the natural transformation swapping the two $V$ parameters.

As explained in the introduction, the definition of the block structure relies on the crucial fact that the functor $(V \Rightarrow-)$ transports $n$-mnemoids (models of $\left.\mathcal{T}_{n}\right)$ to $(n+1)$-mnemoids (models of $\mathcal{T}_{n+1}$ ).

\section{c) A fibrational speculation}

Power shows that the category of models of $\mathcal{T}$ equipped with a block structure is equivalent to the category of algebras of the local state monad [20]. However, we advocate that this block-algebra structure ought to be derived directly from the indexing category of the indexed Lawvere theory $\mathcal{T}$. Let's step back a little and suppose speculatively that we are working with a modified version of the category $I n j$ containing "enough" morphisms to induce the familly of morphisms $\left(\operatorname{block}_{\langle n\rangle}\right)_{n \in \mathbb{N}}$. Let's call $b_{n}$ the (hypothetical) morphism of the indexing category such that

$$
\text { block }_{\langle n\rangle}=A_{b_{n}} \quad: \quad A_{n+1} \quad \longrightarrow \quad A_{n}^{V}
$$

Such an arrow does not correspond to anything existing in the framework of models of indexed Lawvere theories and that is one of our motivations to the fibrational framework introduced in Section III. But for now let's suppose we can work with such morphisms and see what we can deduce from the two preceding diagrams :

$$
\begin{aligned}
A_{b_{n}} \circ A_{i_{n}} & =\text { const }_{A_{n}} \circ A_{i d_{n}} \\
(V \Rightarrow A)_{b_{n}} \circ A_{b_{n+1}} & =\operatorname{swap}^{*} \circ(V \Rightarrow A)_{b_{n}} \circ A_{b_{n+1}} \circ A_{s_{n}^{n+1}}
\end{aligned}
$$

These two equations relate the image of morphisms of the indexing category via some action on the $(V \Rightarrow-)$ functors.
This suggest that the morphisms themselves should be related in some way in the base category :
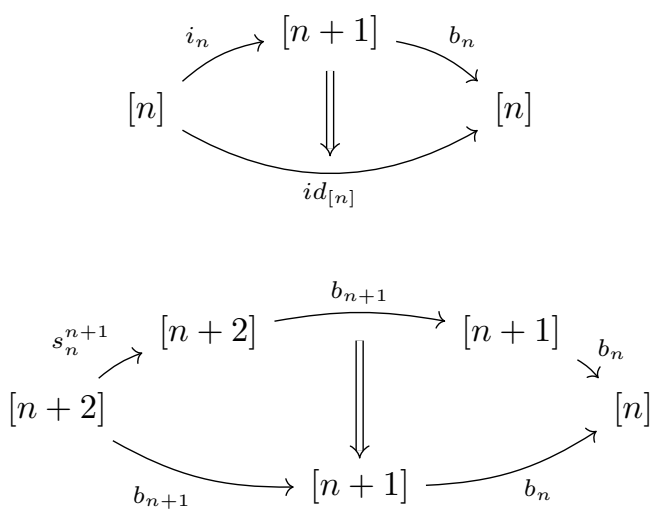

We advocate in the next section that 2-categories are the right setting for obtaining such relations between morphisms.

\section{AN ALGEBRAIC AND 2-CATEGORICAL GROTHENDIECK CONSTRUCTION}

In the preceding section, we outlined how the functor $(V \Rightarrow-)$ enables us to handle allocation by bridging the gap between $n$-mnemoids and $(n+1)$-mnemoids. Thus, the right setting to continue our work should be a category of monads where the we consider monad morphisms acting across some functor via a distributivity law. It turns out that this correspond exactly to the definition given by Street in [10] of the category of monads in $\mathcal{C}$ at. In all this section we work with strict 2categories and strict 2-functors to keep the exposition simple.

\section{a) The 2-category of monads}

First, recall that a monad can be defined in any 2-category $\mathcal{C}$ as a 1-cell $T: c \rightarrow c$ on some object $c \in \mathcal{C}$ equipped with 2-cells

$$
\eta^{T}: I d \longrightarrow T \quad \mu^{T}: T \circ T \longrightarrow T
$$

verifying the usual equations for unit and multiplication. This definition enables one to formally define a 2-category $\mathcal{M n d}(\mathcal{C})$ of monads in $\mathcal{C}$. Since we are only interrested in monads in $\mathcal{C} a t$, we will note $\mathcal{M} n d=\mathcal{M} n d(\mathcal{C} a t)$. The category $\mathcal{M} n d$ has as objects pairs $\langle\mathscr{C}, T\rangle$ of a category $\mathscr{C}$ and a monad $T$ on $\mathscr{C}$. A morphism

$$
\langle F, \lambda\rangle:\left\langle\mathscr{C}_{1}, T_{1}\right\rangle \quad \longrightarrow \quad\left\langle\mathscr{C}_{2}, T_{2}\right\rangle
$$

is then a pair of a functor and a natural transformation

$$
F: \mathscr{C}_{1} \longrightarrow \mathscr{C}_{2} \quad \lambda: T_{2} F \longrightarrow F T_{1}
$$

satisfying the two following properties

$$
\lambda \circ \eta_{F}^{2}=F \eta^{1} \quad \lambda \circ \mu_{F}^{2}=F \mu^{1} \circ \lambda_{T_{1}} \circ T_{2} \lambda
$$



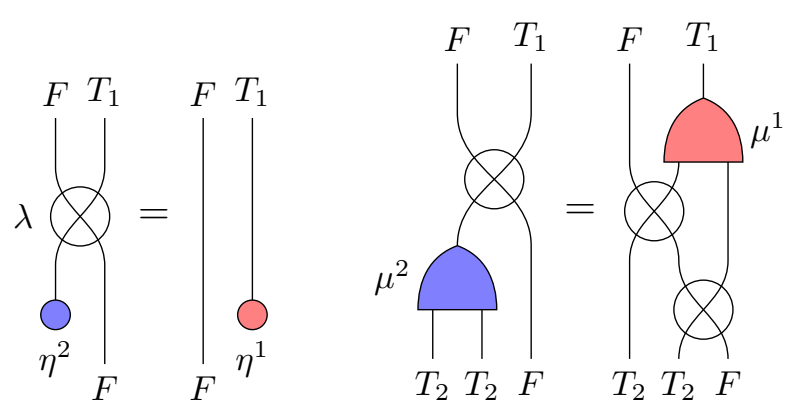

Moreover, 2-cells between morphisms $(F, \lambda)$ and $(G, \nu)$ are natural transformations $\theta: F \rightarrow G$ such that

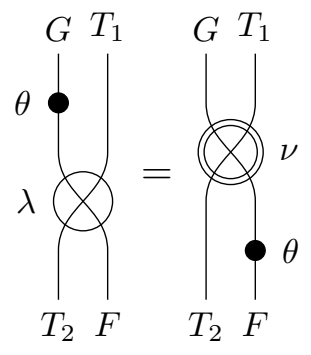

$$
\theta_{T_{1}} \circ \lambda=\nu \circ T_{2} \theta
$$

In particular, $\mathcal{M} n d$ contains objects $\left(S e t, T_{n}\right)$ corresponding to the state monad on $n$ register on Set and morphisms of the form $(V \Rightarrow-, \lambda)$ from $T_{n}$ to $T_{n+1}$ for each $n \in \mathbb{N}$ that we will use extensively in the next section.

\section{b) A 2-Grothendieck construction}

Let us fix some arbitrary 2 -category $\mathcal{B}$ and a 2 -functor

$$
\mathcal{F}: \mathcal{B}^{o p(1,2)} \longrightarrow \mathcal{M} n d
$$

Here $\mathcal{B}^{o p(1,2)}$ denotes the category obtained from $\mathcal{B}$ by formally reversing 1-cells as well as 2-cells. Note that the category $\mathcal{M} n d$ that we introduced in the last section contains a terminal object $*=\left\langle *, i d_{*}\right\rangle$ which consists of the identity monad on the terminal category. In the same way as the original Grothendieck construction, we can use this object * by considering the colax slice category over $\mathcal{F}$ with apex *. This 2-category consists roughly of points over the $\mathcal{B}$ shaped diagram $\mathcal{F}$ and triangles commuting up to natural transformations. In detail, the colax slice category over $\mathcal{F}$ is defined by the following data :

- its objects are pairs $(b,\langle A, \lambda\rangle)$ of an object $b \in \mathcal{B}$ and a 1-cell $\langle A, \lambda\rangle \in \mathcal{M} n d(*, \mathcal{F}(b))$. Posing $\mathcal{F}(b)=\left\langle\mathscr{C}_{b}, T_{b}\right\rangle$, we can identify the functor

$$
A: * \longrightarrow \mathscr{C}_{b}
$$

with a single object $A \in \mathscr{C}_{b}$ and the natural transformation

$$
\lambda: T_{b} A \longrightarrow A
$$

with a $T_{b}$-algebra structure on $A$. Indeed, the two commuting diagrams (6) induce exactly the required equations for an algebra.
- its 1-cells between $(b,\langle A, \alpha\rangle)$ and $\left(b^{\prime},\langle B, \beta\rangle\right)$ are pairs $(f, \varphi)$ of a morphism $f \in \mathcal{B}\left(b, b^{\prime}\right)$ which induces a pair $\mathcal{F}(f)=\left(F_{f}, \lambda_{f}\right)$ and a natural transformation $\varphi: A \rightarrow F_{f} \circ B$
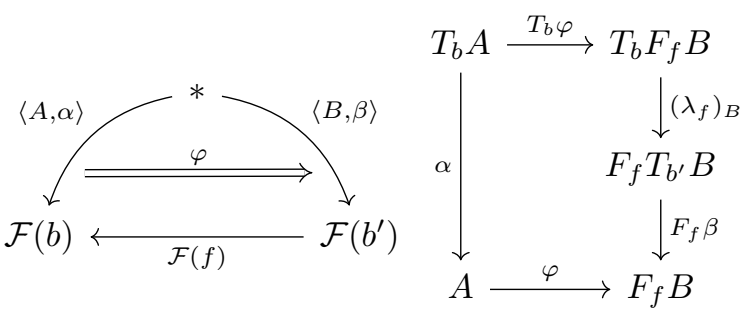

The commuting diagram on the right-hand side obtained from (7) shows that $\varphi$ can be identified with a $T_{b}$-algebra morphism.

- its 2-morphisms between $(f, \varphi)$ and $(g, \psi)$ are 2morphisms $\alpha \in \mathcal{B}(f, g)$ such that

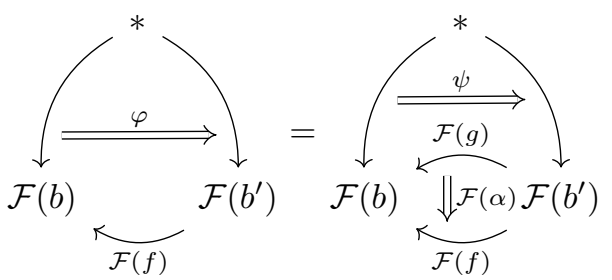

Let us call this 2-category $\int \mathcal{F}$ by analogy with the Grothendieck construction. From the description of $\int \mathcal{F}$, there is an obvious forgetful functor

$$
\pi_{\mathcal{F}}: \int \mathcal{F} \longrightarrow \mathcal{B}
$$

which projects out the first component from objects, morphisms and 2-morphisms onto $\mathcal{B}$. This 2 -functor $\pi_{\mathcal{F}}$ is a 2fibration in the sense of [8].

Indeed, given a 1 -cell in $\mathcal{B}$

$$
f: b^{\prime} \longrightarrow b
$$

and an element $\left\langle b, X_{b}\right\rangle$ in the fiber $\pi_{\mathcal{F}}^{-1}(b)$ of $b$, we have an arrow covering $f$

$$
\varphi=\left\langle f, i d_{F(f) \circ X_{b}}\right\rangle \quad: \quad\left\langle b^{\prime}, F(f) \circ X_{b}\right\rangle \quad \longrightarrow \quad\left\langle b, X_{b}\right\rangle
$$

which is 2 -cartesian, in the sense that the following commuting diagram square is a (strict) pullback square :

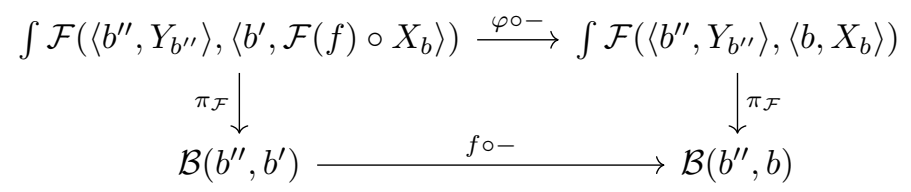

Moreover, the restriction of $\pi_{\mathcal{F}}$ on the $\mathcal{H o m}$-categories are (1-)fibrations and precomposition by a 1-cell of the total space is a morphism of fibration.

Let us note that the fiber over some object $c \in \mathcal{B}$ is the category of $\mathcal{F}(c)$-algebras. Thus, a section of the fibration $\pi_{\mathcal{F}}$, that is a 2 -functor $s: \mathcal{B} \rightarrow \int \mathcal{F}$ such that

$$
s \circ \pi_{\mathcal{F}}=i d_{\mathcal{B}}
$$


picks out a family $s(c)$ of $\mathcal{F}(c)$-algebras indexed by the objects of $\mathcal{B}$ and coherently with respect to the 1-cells and 2-cells present in the base category $\mathcal{B}$.

We will need in the next section a slight extension of this construction working for weak 2-functor $\mathcal{F}: \mathcal{B}^{o p(1,2)} \rightarrow \mathcal{M n d}$ betwteen strict 2-categories $\mathcal{B}$ and $\mathcal{M}$ nd which is presented in Annex VII. It must be noted that even in this case, both the resulting total space and fibration are still strict 2-categories and 2-functors respectively.

\section{THE 2-CATEGORY $\mathcal{P} I n j$}

In this section we instantiate the fibrational framework presented in Section III to obtain a new presentation of the local state monad. In order to do so, we first construct a 2 -category $\mathcal{P} I n j$ of resources which will serve as a base category for the fibration. Then we describe a 2 -functor from this resource category $\mathcal{P} I n j$ into the category of monads $\mathcal{M} n d$.

\section{a) The 2-category of partial injections}

We stressed out in Section II that the ideal category of resources can be thought as completing the category $I n j$ with "missing" arrows. Informally speaking, the category $I n j$ is generated by the two following morphisms

$$
\begin{aligned}
& \text { dealloc : }[0] \quad \longrightarrow[1] \\
& \text { permute : [2] } \longrightarrow[2]
\end{aligned}
$$

We want to add some operation

$$
\text { alloc }:[1] \quad \longrightarrow \quad[0]
$$

with the following 2-cells between morphisms

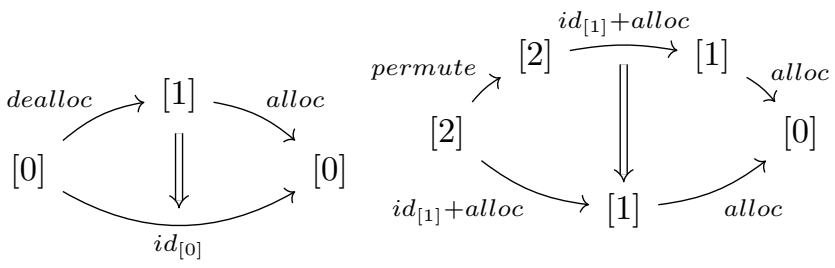

We can obtain this exact setting by considering the category $\mathcal{P} I n j$ of partial injections. Its set of objects consists of finite cardinals $[n]$ for $n \in \mathbb{N}$. A partial injection between $[m]$ and $[n]$ is a pair $\langle p, f\rangle$ of an integer $p \in \mathbb{N}$ and an injection $f:[m] \hookrightarrow[n+p]$. If $\left\langle p^{\prime}, f^{\prime}\right\rangle:[n] \rightarrow[o]$ is another arrow in $\mathcal{P}$ In $j$, composition with $\langle p, f\rangle$ is given by

$$
\left\langle p^{\prime}, f^{\prime}\right\rangle \circ\langle p, f\rangle=\left\langle p^{\prime}+p,\left(g+i d_{p}\right) \circ f\right\rangle:[m] \longrightarrow[o]
$$

Two partial injections $\langle p, f\rangle$ and $\langle q, g\rangle$ between $[m]$ and $[n]$ can be related by an injection $\alpha:[q] \hookrightarrow[p]$ such that

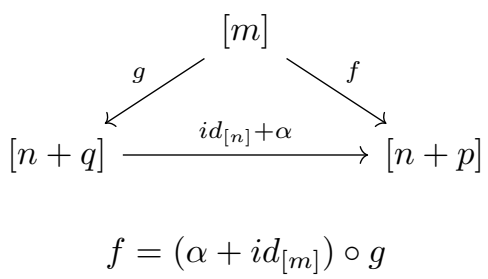

Thus we have a 2-category $\mathcal{P} I n j$ of finite cardinals, partial injections and injections relating the partial parts as 2-cells. In this setting the morphisms dealloc and permute are inherited from $I n j$ and correspond respectively to the pairs

$$
\left\langle 0, i_{0}\right\rangle:[0] \rightarrow[1] \quad\left\langle 0, s_{0}^{1}\right\rangle:[2] \rightarrow[2]
$$

where $i_{0}$ is the only application from the empty set to the singleton and $s_{0}^{1}$ permute the two elements of [2].

The morphism alloc is given by the pair $\left\langle 1, i d_{[1]}\right\rangle:[1] \rightarrow[0]$. Then we can compute that we have the following 2-cells :

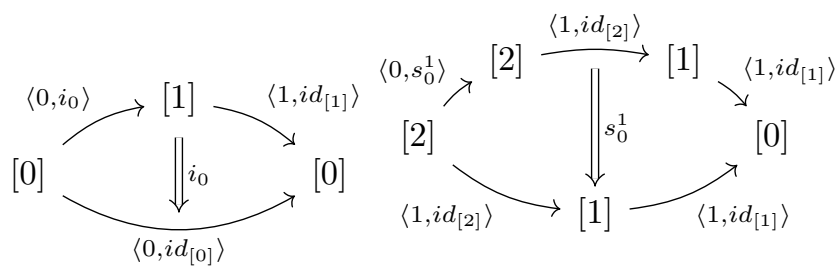

\section{b) A 2-functor into monads.}

We will turn now to the definition of the 2-functor

$$
\mathscr{T} \quad: \mathcal{P I n j}^{\mathrm{op}(1,2)} \longrightarrow \mathcal{M} \text { nd }
$$

We naturally set $\mathcal{T}([n])$ to be the pair $\left\langle\right.$ Set, $\left.T_{n}\right\rangle$ corresponding to the state monad on $n$ registers $T_{n}:$ Set $\rightarrow$ Set. Then given a partial injection

$$
\langle p, f\rangle \quad: \quad[m] \quad \longrightarrow \quad[n]
$$

we have to describe a morphism $T_{m} \rightarrow T_{n}$, that is a pair of an endofunctor $F$ on Set and a natural transformation $\lambda^{\langle p, f\rangle}: T_{m} F \rightarrow F T_{n}$. Since we observed earlier that the functor $(V \Rightarrow-)$ allowed us to move from $T_{n}$ to $T_{n+1}$, we set $F=\left(V^{p} \Rightarrow-\right)$ and construct the following natural transformation

$$
\lambda_{A}^{\langle p, f\rangle}: V^{m} \Rightarrow\left(V^{p} \Rightarrow A\right) \times V^{m} \longrightarrow V^{p} \Rightarrow V^{n} \Rightarrow A \times V^{n}
$$

Let $A$ be a set, $h \in V^{m} \Rightarrow\left(V^{p} \Rightarrow A\right) \times V^{m}$ and $v^{p} \in V^{p}$, $v^{n} \in V^{n}$ be vectors of values. We must construct an object

$$
\lambda_{A}^{\langle p, f\rangle}(f)\left(v^{p}\right)\left(v^{n}\right) \quad \in A \times V^{n}
$$

First note that the concatenation of $v^{p}$ and $v^{n}$ gives us a vector $\left(v^{n} \cdot v^{p}\right) \in V^{n+p}$. Pulling back that vector along $f$ gives us an element in $V^{m}$ on which we can evaluate $h$ yielding a pair :

$$
\left(h_{0}, v^{m}\right)=h \circ f^{*}\left(v^{n} \cdot v^{p}\right) \in\left(V^{p} \Rightarrow A\right) \times V^{m}
$$

Now, evaluating the first component on $v^{p}$ gives us an element $h_{0}\left(v^{p}\right) \in A$. The final piece we need is a vector of values in $v^{n}$. It is obtained by first rewriting $\left(v^{n} \cdot v^{p}\right)$ by pushing forward $v^{m}$ along $f$ and then projecting on the $n$ first components of the vector via $\pi_{n}^{n+p}: V^{n+p} \rightarrow V^{n}$. More formally, for $v \in V^{m}$ and $w \in V^{n+p}$, we introduce an operation $f_{*}^{w}$ : $V^{m} \rightarrow V^{n+p}$

$$
\left(f_{*}^{w}(v)\right)(i)= \begin{cases}v_{j} & \text { if }\{j\}=f^{-1}(i) \\ w_{i} & \text { otherwise }\end{cases}
$$


and we finally get as result for $\lambda_{A}^{\langle p, f\rangle}(f)\left(v^{p}\right)\left(v^{n}\right)$

$$
\left(h_{0}\left(v^{p}\right), \pi_{p}^{n+p} \circ f_{*}^{\left(v^{n} \cdot v^{p}\right)}\left(v^{m}\right)\right) \in A \times V^{n}
$$

Putting everything together gives us the following definition for $\lambda^{\langle p, f\rangle}$ :

$$
h \mapsto \lambda v^{p} \cdot \lambda v^{n} \cdot\left(e v a l_{v^{p}} \times \pi_{n}^{n+q} \circ f_{*}^{\left(v^{n} \cdot v^{p}\right)}\right) \circ h \circ f^{*}\left(v^{n} \cdot v^{p}\right)
$$

Finally, given a 2-cell

$$
\alpha:\langle p, f\rangle \longrightarrow\langle q, g\rangle:[m] \longrightarrow[n]
$$

that is an injection $\alpha:[q] \hookrightarrow[p]$ such that $f=\left(i d_{[n]}+\alpha\right) \circ g$, we must describe a natural transformation

$$
\theta \quad: \quad\left(V^{q} \Rightarrow-\right) \quad \longrightarrow \quad\left(V^{p} \Rightarrow-\right)
$$

satisfying (7). The operation $V^{\alpha}$ of pulling back along $\alpha$ sends an element of $V^{p}$ to $V^{q}$ and precomposing by this map gives the desired natural transformation $\theta=\left(-\circ V^{\alpha}\right)$. The required equation (7) comes from the fact that pulling back along $f$ is the same as pulling back along $\left(i d_{[n]}+\alpha\right) \circ g$, that is first pulling back along $\alpha$ and then along $g$. It can be noted that we do not obtain a strict 2-functor but only a weak 2 -functor $\mathscr{T}$. Indeed, we have a natural isomorphism

$$
\left(V^{n} \Rightarrow\left(V^{m} \Rightarrow-\right)\right) \quad \longrightarrow \quad\left(V^{n+m} \Rightarrow-\right)
$$

instead of an equality for each $n, m \in \mathbb{N}$. It can be checked that this family of natural isomorphism induces the required structure on $\mathcal{T}$ in order to have a weak 2 -functor.

\section{c) The fibration over $\mathcal{P}$ In $j$}

We described in Section III a way to construct a fibration over a base $\mathcal{B}$ given a 2 -functor $\mathcal{B} \rightarrow \mathcal{M}$ nd. Even though we only considered there the case of a strict 2-functor, Annex VII shows that the construction does extend to the weak case and that the resulting total category is still a strict 2-category. As such, let us unroll the definition of the category $\int \mathscr{T}$ built from the 2 -functor $\mathscr{T}$ introduced in the previous section.

Its objects are pairs of a natural number $n$ and an algebras of the global state monad on $n$ registers. A morphism between a $T_{n}$-algebra $(A, \alpha)$ and a $T_{m}$-algebra $(B, \beta)$ is a pair consisting of a partial injection $(p, f):[n] \rightarrow[m]$ and a $T_{n}$-algebra morphism $A \rightarrow V^{p} \Rightarrow B$. The $T_{n}$-algebra structure on $V^{p} \Rightarrow$ $B$ is obtained by pulling back the $T_{m}$-algebra structure of $B$ along $f$ :

$$
T_{n}\left(V^{p} \Rightarrow B\right) \stackrel{\lambda_{B}^{(p, f)}}{\longrightarrow} V^{p} \Rightarrow T_{m}(B) \stackrel{V^{p} \Rightarrow \beta}{\longrightarrow} V^{p} \Rightarrow B
$$

Moreover, if $\varphi: A \rightarrow V^{p} \Rightarrow B$ and $\psi: A \rightarrow V^{q} \Rightarrow B$ are $T_{n}$-algebra morphisms respectively over $(p, f)$ and $(q, g)$, the 2 -cells between them are given by the 2-cells between $(p, f)$ and $(q, g)$ in $\mathcal{P} I n j$, that is injections $\alpha:[q] \hookrightarrow[p]$ such that $f=\left(i d_{[n]}+\alpha\right) \circ g$ inducing an equation $\varphi=\left(-\circ V^{\alpha}\right) \circ \psi$.

From this description, the fibration $\pi_{\mathscr{T}}: \int \mathscr{T} \rightarrow \mathcal{P}$ Inj sends a $T_{n}$-algebra to the cardinal $[n]$, a morphism in $\int \mathscr{T}$ to the partial injection present as its first component and a 2-morphism to itself.

\section{d) Algebras as sections}

Now, we can consider the category of sections $\Gamma\left(\pi_{\mathscr{T}}\right)$ of the fibration $\pi_{\mathscr{T}}$, that is the full subcategory of 2-functors $F$ from $\mathcal{P} I n j$ to $\int \mathscr{T}$ such that $\pi_{\mathscr{T}} \circ F=i d_{\mathcal{P} I n j}$. An object $\mathscr{S} \in \Gamma\left(\pi_{\mathscr{T}}\right)$ consist of the following data :

- a family $\left(A_{n}\right)_{n \in \mathbb{N}}$ of sets where $\mathscr{S}(n)=A_{n}$ is endowed with a $T_{n}$-algebra structure

- for each natural number $p$ and injection $f:[m] \hookrightarrow[n+p]$, a $T_{m}$-algebra morphism

$$
\varphi_{\langle p, f\rangle} \quad: \quad A_{m} \quad \longrightarrow \quad V^{p} \Rightarrow A_{n}
$$

- such that any 2 -cell, that is any equation $f=\left(i d_{n}+\alpha\right) \circ g$, induces a 2-cell in the total space, that is a commutative diagram

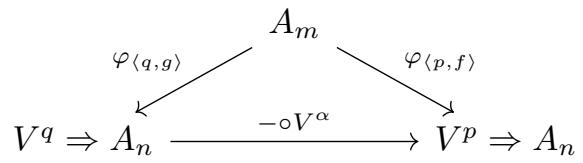

We will now show that this category $\Gamma\left(\pi_{\mathscr{T}}\right)$ of sections is equivalent to the category of models of the indexed Lawvere theory $\mathcal{T}$ equipped with a block-algebra structure as introduced by Power. This will suffice to prove our claim that the algebras of the local state monad can be considered as the aforementioned sections.

Let's begin by noting that there is an obvious inclusion functor

$$
\mathcal{J} \quad: \quad \operatorname{Inj} \quad \longrightarrow \mathcal{P} \text { Inj }
$$

sending each natural number to itself and an injection $f$ to the pair $\langle 0, f\rangle$. Also recall that for any natural number $n$, the category of $n$-mnemoids coincides with both the category $\mathcal{T}_{n}$ Mod of models of the Lawvere theory $\mathcal{T}_{n}$ of state on $n$ registers and the category $T_{n}$-Alg of algebra of the state monad $T_{n}$ on $n$ registers.

Given a section $\mathscr{S} \in \Gamma\left(\pi_{\mathscr{T}}\right)$, we can precompose it with $\mathcal{J}$ and consider it as taking its value in $\mathcal{T}_{n}$-Mod instead of $T_{n}$-alg, thus yielding a model $\mathcal{M}_{\mathscr{S}}$ of the indexed Lawvere theory of local state $\mathcal{T}$. Moreover, we can equip $\mathcal{M}_{\mathscr{S}}$ with a block-algebra structure by setting for each natural number $n \in \mathbb{N}$

$$
\mathrm{block}_{n}=\mathscr{S}\left\langle 1, i d_{n+1}\right\rangle \quad: \quad A_{n+1} \quad \longrightarrow \quad V \Rightarrow A_{n}
$$

The two required commutating diagrams (4) and (5) for the block-algebra structure are respectively the image by $\mathscr{S}$ of the following 2-cells

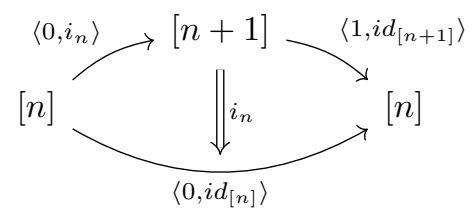




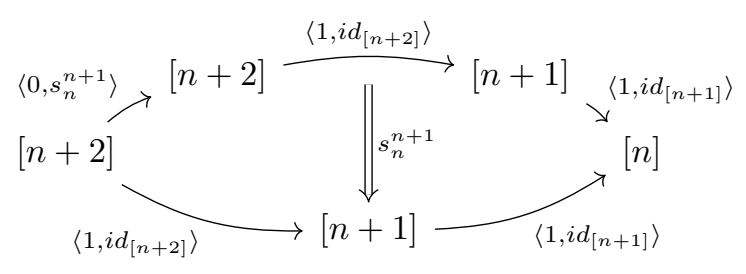

Reciprocally, we can build a section $\mathscr{S}_{\mathcal{M}}$ from a model $\mathcal{M}$ of the indexed Lawvere theory for local state $\mathcal{T}$ equipped with a block-algebra structure $\left(\text { block }_{n}\right)_{n \in \mathbb{N}}$. For an object $[n] \in$ $\mathcal{P} I n j$, we set $\mathscr{S}_{\mathcal{M}}(n)$ to be $\mathcal{M}_{n}$ now seen as a $T_{n}$-algebra. Then for a partial injection

$$
\langle p, f\rangle \quad: \quad[m] \quad \longrightarrow \quad[n]
$$

we set $\mathscr{S}_{\mathcal{M}}\langle p, f\rangle$ to be the following composite

$$
\mathscr{S}_{\mathcal{M}}(m) \stackrel{\mathcal{M}_{f}}{\longrightarrow} \mathscr{S}_{\mathcal{M}}(n+p) \stackrel{\operatorname{block}_{n}^{p}}{\longrightarrow} \quad V^{p} \Rightarrow \mathscr{S}_{\mathcal{M}}(n)
$$

where the right arrow $\mathrm{block}_{n}^{p}$ is the following composite

$$
\left(V^{p-1} \Rightarrow \operatorname{block}_{\langle n\rangle}\right) \circ \cdots \circ\left(V \Rightarrow \operatorname{block}_{\langle n+p-1\rangle}\right) \text { block }_{\langle n+p\rangle}
$$

It can be checked that $\mathscr{S}_{M}\langle p, f\rangle$ is a $T_{m}$-algebra morphism as needed.

Finally, we must show that any 2-cell in the base

$$
\alpha:\langle p, f\rangle \longrightarrow\langle q, g\rangle:[m] \longrightarrow[n]
$$

induces a commuting diagram in the total space :

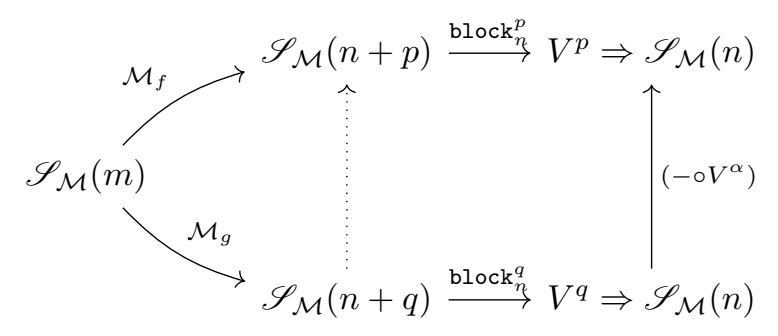

It is proved by first instanciating the dotted arrow by $\mathcal{M}_{i d_{[n]}+\alpha}$ and then proving separately that the left triangle and right square commute.

For the left triangle, the definition of the 2 -cell $\alpha$ give us

$$
f=\left(i d_{[n]}+\alpha\right) \circ g
$$

and by functoriality of $\mathcal{M}$ we get

$$
\mathcal{M}_{f}=\mathcal{M}_{i d_{[n]}+\alpha} \circ \mathcal{M}_{g}
$$

For the right square, first recall that the monoidal category $I n j$ is generated by the operations $i_{0}:[0] \rightarrow[1]$ and $s_{0}^{1}:[2] \rightarrow[2]$. Given a term presenting $\alpha$ built upon these two operations, we can easily prove by structural induction on the term that the square commutes, using the diagrams (4) and (5) in the base case.

It is not difficult to see that the two transformations that we just described are inverse of each other and that they extends straightforwadly to morphisms. Hence we obtain the following :
Theorem 1. The category $\Gamma\left(\pi_{\mathscr{T}}\right)$ of sections of the fibration $\pi_{\mathscr{T}}$ is equivalent to the category of models of the indexed Lawvere theory $\mathcal{T}$ equipped with a block algebra structure.

Thus an algebra of the local state monad can be seen as well as a section of $\pi_{\mathscr{T}}$, since the former is proved by Power to be equivalent to a model of $\mathcal{T}$ equipped with a blockalgebra structure. However, the proof of equivalence relies on Beck's monadicity theorem which prevent us from having a conceptual overview of the variations between the different presentations of the local state monad.

\section{Connection with the Algebraic PRESEntation}

We exhibit in this section a direct proof of isomorphism between our category of sections $\Gamma\left(\pi_{\mathscr{T}}\right)$ and the category of algebras of the local state monad $T$ on $[I n j, S e t]$. Due to the complicated nature of the local state monad $T$ on $[$ Inj, Set $]$, we chose to introduce here the presentation given by Melliès in [12] of the local state monad $T_{\text {Res }}$ on $[R e s, S e t]$ where Res is a category obtained from Inj by adding some morphisms as explained thereafter.

Indeed, given a covariant presheaf on Res

$$
A \quad: \text { Res } \longrightarrow \text { Set }
$$

the local state monad $T_{\text {Res }}$ takes the following particularly nice expression on objects :

$$
T_{\text {Res }} A \quad: \quad[n] \quad \longmapsto T_{n} A_{n}=V^{n} \Rightarrow A_{n} \times V^{n}
$$

That is the local state monad $T_{\text {Res }}$ coincides pointwise with the state monad $T_{n}$ on $n$ registers.

\section{a) The category Res of resources}

The category Res of resources has natural number as objects and resource morphisms as 1-cells. A resource morphism $f$ between $[m]$ and $[n]$ is defined as a function

$$
f \quad: \quad[m] \quad \longrightarrow \quad[n] \uplus V
$$

where every element in $[n]$ has at most one antecedent. As such, any injection $f:[m] \rightarrow[n]$ can be seen as a resource morphism and thus there is an induced embedding

$$
\iota: \text { Inj } \longrightarrow \text { Res }
$$

sending each object to itself and coercing transparently injections to resource morphisms.

There is another description of resource morphisms as equivalence class which may seem less natural but will simplify greatly the presentation of the connection to $\Gamma\left(\pi_{\mathscr{T}}\right)$. The idea is that a resource morphism can be seen as a pair of an injection and a vector of values up to some equivalence relation relating pairs sending the same inputs to the same outputs. This can be expressed formally with the following coend formula :

$$
\operatorname{Res}(m, n) \cong \int^{p \in \operatorname{Inj}} \operatorname{Inj}(m, n+p) \times V^{p}
$$

That is a resource morphism from $[m]$ to $[n]$ is a pair $[f, v]_{p}$ composed of an injection $f:[m] \rightarrow[n+p]$ and a vector of 
values $v \in v^{p}$. Moreover two such pairs $[f, v]_{p_{1}}$ and $[g, w]_{p_{2}}$ are identified when there exists an injection $\alpha:\left[p_{1}\right] \rightarrow\left[p_{2}\right]$ such that :

$$
\left(i d_{n}+\alpha\right) \circ f=g \quad v=V^{\alpha}(w)
$$

This reformulation of resource morphisms enable us to give a concise description of pull back and push forward operations for resources morphisms in terms of the very same operations for injections. Recall that we introduced and used the operations of pulling back a state along an injection and updating a previous state by pushing forward a newer but smaller state along an injection in (9). Given a resource morphism $\mathbf{f}:[m] \rightarrow[n]$ and a piece of state $v^{n} \in V^{n}$, we hence have the two following operations :

$$
\begin{array}{rllll}
\mathbf{f}^{*} & : & V^{n} & \longrightarrow & V^{m} \\
\mathbf{f}_{*}^{v_{n}} & : & V^{m} & \longrightarrow & V^{n}
\end{array}
$$

Moreover, if $\mathbf{f}$ is represented by the pair $\left[f, v^{p}\right]_{p}$ of an injection $f:[m] \rightarrow[n+p]$ and a vector of values $v^{p} \in V^{p}$, then we have the following definitions :

$$
\begin{aligned}
{\left[f, v_{p}\right]^{*}\left(v_{n}\right) } & =f^{*}\left(v_{n} \cdot v_{p}\right) \\
{\left[f, v_{p}\right]_{*}^{v_{n}}\left(v_{m}\right) } & =f_{*}^{\left(v_{n} \cdot v_{p}\right)}\left(v_{m}\right)
\end{aligned}
$$

It is not difficult to calculate that these definitions do not depend on the choice of the representative taken for $f$.

\section{b) A local state monad on $[$ Res, Set $]$}

The construction of the local state monad $T_{R e s}$ on [Res, Set] starts with the observation that the local state monad $T$ on $[$ Inj, Set $]$ can be decomposed as the composition of a change of base monad $\mathscr{B}$ and a fiber monad $\mathscr{F}$ regulated by a distributivity law

$$
\lambda: \mathscr{B} \circ \mathscr{F} \longrightarrow \mathscr{F} \circ \mathscr{B}
$$

where the change of base monad $\mathscr{B}$ is obtained from the following adjunction

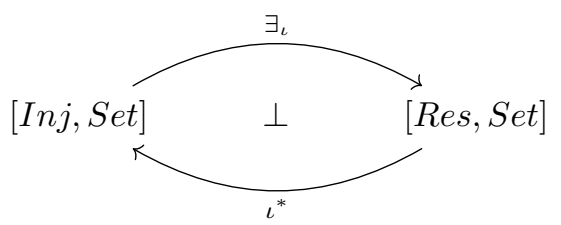

From the general theory of distributivity laws [1], it then follows that the fiber monad $\mathscr{F}$ can be lifted to a monad $T_{\text {Res }}$ on $[R e s, S e t]$ whose algebras coincides with the algebras of the local state monad on $[$ Inj, Set] , hence the name of local state monad on $[$ Res, Set $]$ for $T_{\text {Res }}$.

As we announced at the beginning of this section, the monad $T_{R e s}$ act as follows on a covariant presheaf $F \in[$ Res, Set $]$

$$
T_{\text {Res }} F \quad: \quad[n] \quad \longmapsto T_{n}(F(n))
$$

Then, if $\mathbf{f}:[m] \rightarrow[n]$ is a resource morphism, we have the following definition of $T_{R e s} F$ on morphisms

$$
T_{R e s} F(\mathbf{f}):\left\{\begin{array}{c}
V^{m} \Rightarrow F(m) \times V^{m} \longrightarrow V^{n} \Rightarrow F(n) \times V^{n} \\
h \quad \longmapsto \lambda v \cdot\left(F(\mathbf{f}) \times \mathbf{f}_{*}^{v}\right) \circ h \circ \mathbf{f}^{*}(v)
\end{array}\right.
$$

The action of the monad $T_{\text {Res }}$ on a natural transformation $\theta=\left(\theta_{n}\right)_{n \in \mathbb{N}}$ is simply to apply the relevant state monad $T_{n}$ on $n$ registers, that is $\left(T_{R e s} \theta\right)_{n}=T_{n} \theta_{n}$. The unit and multiplication are also given pointwise by those of $T_{n}$ for each natural number $n$.

\section{c) A second proof of equivalence}

Now we turn to the proof of the following theorem :

Theorem 2. The category $T_{r e s}$-Alg of the algebras of the local state monad $T_{\text {Res }}$ on $[R e s, S e t]$ is equivalent to the category $\Gamma\left(\pi_{\mathscr{T}}\right)$ of sections of the fibration $\pi_{\mathscr{T}}$.

Given an object in $T_{R e s}$-alg, that is a pair of a covariant presheaf $F$ and an algebra map $\alpha$

$$
F: \text { Res } \longrightarrow \text { Set } \quad \alpha: T_{\text {Res }} F \longrightarrow F
$$

we construct a section $\mathscr{S}$ as follows. On an object of $\mathcal{P} I n j$, that is a finite cardinal $[n]$, we set $\mathscr{S}(n)$ to be the $T_{n}$-algebra $\left\langle F(n), \alpha_{n}\right\rangle$. Then we need to define $\mathscr{S}$ on a partial injection

$$
\langle p, f\rangle \quad: \quad[m] \quad \longrightarrow \quad[n]
$$

In order to do so, first note that we have an operation

$$
F\left([f,-]_{p}\right) \quad: \quad V^{p} \times F(m) \quad \longrightarrow \quad F(n)
$$

Hence, we give the following definition for $\mathscr{S}(f)$

$$
\begin{aligned}
& \mathscr{S}(m) \quad \longrightarrow \quad V^{p} \Rightarrow \mathscr{S}(n) \\
& h \quad \longmapsto \quad \lambda v^{p} \cdot F\left(\left[f, v^{p}\right]_{p}\right)(h)
\end{aligned}
$$

We ought to check that this function is indeed a $T_{m}$-algebra morphism. This can be derived from the following commuting diagram :

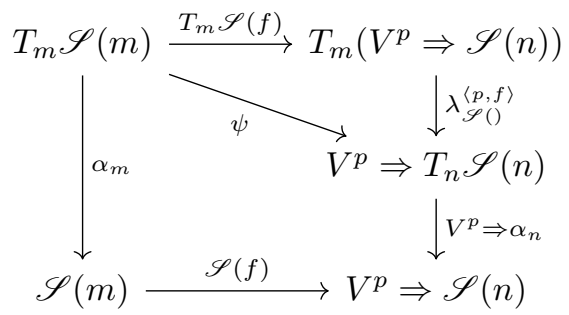

where $\psi=h \mapsto \lambda v^{p} . T_{\text {Res }} F\left(\left[f, v^{p}\right]\right)(h)$. The lower rectangle commutes because $F$ is a $T_{R e s}$-algebra and the commutation of the upper triangle follows from a little calculation.

Finally, given a 2 -cell in $\mathcal{P} I n j$

$$
\alpha:\langle p, f\rangle \longrightarrow\langle q, g\rangle:[m] \longrightarrow[n]
$$

we must check that $\mathscr{S}(f)=\left(-\circ V^{\alpha}\right) \circ \mathscr{S}(g)$, which comes from the next calculation where $h \in \mathscr{S}(m)$ :

$$
\begin{aligned}
\left(-\circ V^{\alpha}\right) \circ \mathscr{S}(g)(h) & =\left(-\circ V^{\alpha}\right)\left(\lambda v^{q} \cdot F\left(\left[g, v^{q}\right]_{q}\right)(h)\right) \\
& =\lambda v^{p} \cdot F\left(\left[g, V^{\alpha}\left(v^{p}\right)\right]_{q}\right)(h) \\
& =\lambda v^{p} \cdot F\left(\left[\left(i d_{[n]}+\alpha\right) \circ g, v^{p}\right]_{p}\right)(h) \\
& =\lambda v^{p} \cdot F\left(\left[f, v^{p}\right]_{p}\right)(h)=\mathscr{S}(f)(h)
\end{aligned}
$$

Reciprocally, starting from a section $\mathscr{S} \in \Gamma\left(\pi_{\mathscr{T}}\right)$, we construct a covariant presheaf $F \in[R e s$, Set $]$ and equip it 
with a $T_{R e s}$-algebra structure. On an object $[n] \in R e s$, we set $F(n)=A_{n}$ to be the set underlying the $T_{n}$-algebra $\mathscr{S}(n)=$ $\left\langle A_{n}, \alpha_{n}\right\rangle$. Then given a resource morphism $\mathbf{f} \in \operatorname{Res}([m],[n])$ represented by a pair $[f, v]_{p}$

$$
f \quad: \quad[m] \longrightarrow[n+p] \quad v^{p} \in V^{p}
$$

we can take

$$
\begin{aligned}
F(\mathbf{f}): \quad & F(m) \\
& \longrightarrow \quad F(n) \\
h & \longmapsto \mathscr{S}\langle p, f\rangle(h)\left(v^{p}\right)
\end{aligned}
$$

This definition does not depend on the choice of the representative, because any other representative $\mathbf{f}=\left[g, v^{q}\right]_{q}$ will be related to the first by an injection $\alpha$ which can be seen as a 2-cell in $\mathcal{P}$ Inj

$$
\alpha \quad: \quad\langle p, f\rangle \longrightarrow\langle q, g\rangle \quad: \quad[m] \longrightarrow[n]
$$

from which follows with a little calculation that for any $h \in F(m)$

$$
\mathscr{S}\langle p, f\rangle(h)\left(v^{p}\right)=\mathscr{S}\langle q, g\rangle(h)\left(v_{q}\right)
$$

The $T_{R e s}$-algebra map on $F$ is then taken as the family $\left(\alpha_{n}\right)_{n \in \mathbb{N}}$ of maps, where $\alpha_{n}: T_{n} A_{n} \rightarrow A_{n}$ is a $T_{n}$-algebra map.

\section{CONCLUSION}

In this paper, we develop one step further the careful algebraic analysis of the local state monad initiated fifteen years ago with the seminal work by Plotkin and Power. To that purpose, we benefit from the deep connection (which we also clarify in the process) between the notion of indexed Lawvere theories formulated by Power and the algebraic presentations of the local state monad performed by Staton and Melliès. This unified point of view leads us to a purely conceptual description of the local state monad $T$ as the result of "gluing together" a family of global state monads $\left(T_{n}\right)_{n \in \mathbb{N}}$. The glueing itself is performed by applying a Grothendieck construction on an adapted notion of $\mathcal{C}$-indexed monad, simply defined as a 2-functor from $\mathcal{C}^{o p}$ to Street's 2-category $M n d$ of monads. In this 2-categorical way, we are able to see for the first time an algebra $A$ of the local state monad $T$ as a section $\left(A_{n}\right)_{n \in \mathbb{N}}$ of a specific notion of fibration, whose fibers are the categories of $T_{n}$-algebras.

One main purpose of future research will be to combine this fibrational description of the local state monad with the recent work on the proof-relevant semantics of local effects designed by Benton, Hofmann and Nigam [2]. To that purpose, one needs to understand how the shift from discrete fibrations to general 2-fibrations performed here interacts (by the appropriate sheaf or descent conditions) with the setoidal notion of fibration formulated in [2]. This would enable one to reformulate their monadic description as a local and purely equational account, which we believe is a useful step towards formalization in proof assistants.

Another research direction will be to describe other "local effects". We are specifically interested in a notion of heap monad reflecting the behaviour of registers with self reference and pointers. Our fibrational description of the "local heap monad" should bridge the gap with more direct accounts of heaps like separation logic. This investigation is interestingly related to the fibrational account of refinement types recently developed by Melliès and Zeilberger [14].

\section{ACKNOWLEGMENTS}

The authors are grateful to Noam Zeilberger for nice and enlightning discussion during the preparation of this paper.

\section{REFERENCES}

[1] J. Beck, "Distributive laws", in Lecture Notes in Mathematics, vol 80, pp. 119-140, 1969.

[2] N. Benton, M. Hofmann and V. Nigam, "Abstract effects and proofrelevant logical relations", in POPL 2014, pp. 619-632, 2014.

[3] N. Benton, J. Hughes, and E. Moggi, "Monads and effects," in APPSEM Summer School, ser. Lecture Notes In Computer Science, vol. 2395. Springer, 2000, pp. 24-122.

[4] M. Gabbay and A. M. Pitts, "A new approach to abstract syntax involving binders," in Proc. LICS 99. IEEE Press, 1992, pp. 214-224.

[5] J. M. E. Hyland, P. B. Levy, G. Plotkin, and A. J. Power, "Combining algebraic effects with continuations," Theoretical Computer Science, vol. 375, 2007.

[6] J. M. E. Hyland, G. Plotkin, and A. J. Power, "Combining effects: sum and tensor," Theoretical Computer Science, vol. 357, no. 1, pp. 70-99, 2006.

[7] J. M. E. Hyland and A. J. Power, "Discrete lawvere theories and computational effects," Theoretical Computer Science, vol. 366, pp. 144-162, 2006.

[8] C. Hermida, "Descent on 2-fibrations and 2-regular 2-categories", in Applied Categorical Structures, vol. 12, pp. 427-459, 2004.

[9] M. Kelly and R. Street, "Review of the elements of 2-categories," Lecture Notes in Mathematics, vol. 420, pp. 75-103, 1974.

[10] R. Street, "The formal theory of monads", Journal of Pure and Applied Algebra, vol. 2, pp. 149-168, 1972.

[11] S. Lack and R. Street, "The formal theory of monads II," Journal of Pure and Applied Algebra, vol. 175, pp. 243-265, 2002.

[12] P.A. Melliès, "Local States in String Diagrams", in RTA-TLCA 2014, pp. 334-348, 2014.

[13] P.A. Melliès, "Segal Condition Meets Computational Effects", in LICS 2010, pp. 150-159, 2010.

[14] P.A. Melliès and N. Zeilberger, "Functors are Type Refinement Systems", in POPL 2015, pp. 3-16, 2015.

[15] E. Moggi, "Notions of computation and monads," Information And Computation, vol. 93, no. 1, 1991.

[16] K. Nishizawa and A. J. Power, "Lawvere theories enriched over a general base," Journal of Pure and Applied Algebra, vol. 213, pp. 377-386, 2009.

[17] F. J. Oles, "Functor categories and Store shapes", Algol-like languages, vol. 2, pp. 3-12, 1997.

[18] A. M. Pitts, "Nominal Sets: Names and Symmetry in Computer Science", Cambridge Tracts in Theoretical Computer Science, vol. 57, 2013.

[19] G. D. Plotkin and A. J. Power, "Notions of computation determine monads," in Proc. FOSSACS 2002, Lecture Notes in Computer Science, vol. 2303. Springer Verlag, 2002.

[20] J. Power, "Indexed Lawvere theories for local state", CRM Proceedings \& Lectures Notes, vol. 53, pp. 213-229, 2011.

[21] J. Power, "Enriched lawvere theories," Theory and Applications of Categories, vol. 6, no. 7, pp. 83-93, 1999.

[22] P.W. O'Hearn and D. J. Pym, "The logic of bunched implications", Bulletin of Symbolic Logic, vol. 5, pp. 215-244, 1999.

[23] J. C. Reynolds, "Using functor categories to generate intermediate code", Algol-like languages, vol. 2, pp. 13-37, 1997. 
[24] J. C. Reynolds, "Separation Logic: A Logic for Shared Mutable Data Structures", in Proc. LICS 2002, pp. 55-74, 2002.

[25] S. Staton, "Completeness for Algebraic Theories of Local State", in FOSSACS 2010, pp. 48-63, 2010.

[26] M. Weber, "Familial 2-functors and parametric right adjoints," Theory and Applications of Categories, vol. 18, pp. 665-732, 2007.

\section{2-GROTHENDIECK CONSTRUCTION ON A WEAK} 2-FUNCTOR

In this section, we extend our Grothendieck construction of Section III to a weak 2-functor

$$
\mathcal{F}: \mathcal{B}^{o p(1,2)} \longrightarrow \mathcal{M n d}
$$

where $\mathcal{B}$ is an arbitrary strict 2 -category.

Recall that by a weak 2-functor $\mathcal{G}: \mathcal{C} \rightarrow \mathcal{D}$ between strict 2-categories $\mathcal{C}$ and $\mathcal{D}$, we mean that $\mathcal{G}$ consists of the following data :

- an object $\mathcal{G}(c)$ of $\mathcal{D}$ for each object $c \in \mathcal{C}$

- a functor $\mathcal{G}_{c, c^{\prime}}: \mathcal{C}\left(c, c^{\prime}\right) \longrightarrow \mathcal{D}\left(\mathcal{G}(c), \mathcal{G}\left(c^{\prime}\right)\right)$ for each pair of objects $c, c^{\prime} \in \mathcal{C}$

- a family of invertible 2-cells $m_{c}^{0}: i d_{\mathcal{G}(c)} \longrightarrow \mathcal{G}_{c, c}\left(i d_{c}\right)$ in $\mathcal{D}$

- a family of isomorphisms $m_{g, f}^{2}: \mathcal{G}_{c^{\prime}, c^{\prime \prime}}(g) \circ \mathcal{G}_{c, c^{\prime}}(f) \longrightarrow$ $\mathcal{G}_{c, c^{\prime \prime}}(g \circ f)$ in $\mathcal{D}$ natural in $f$ and $g$ for any pair of composable arrows $f \in \mathcal{C}\left(c, c^{\prime}\right)$ and $g \in \mathcal{C}\left(c^{\prime}, c^{\prime \prime}\right)$.

- Associativity and unitality axioms where the functors $\mathcal{G}_{c, c^{\prime}}$ on Hom-categories have been abbreviated by $\mathcal{G}$ for readability :
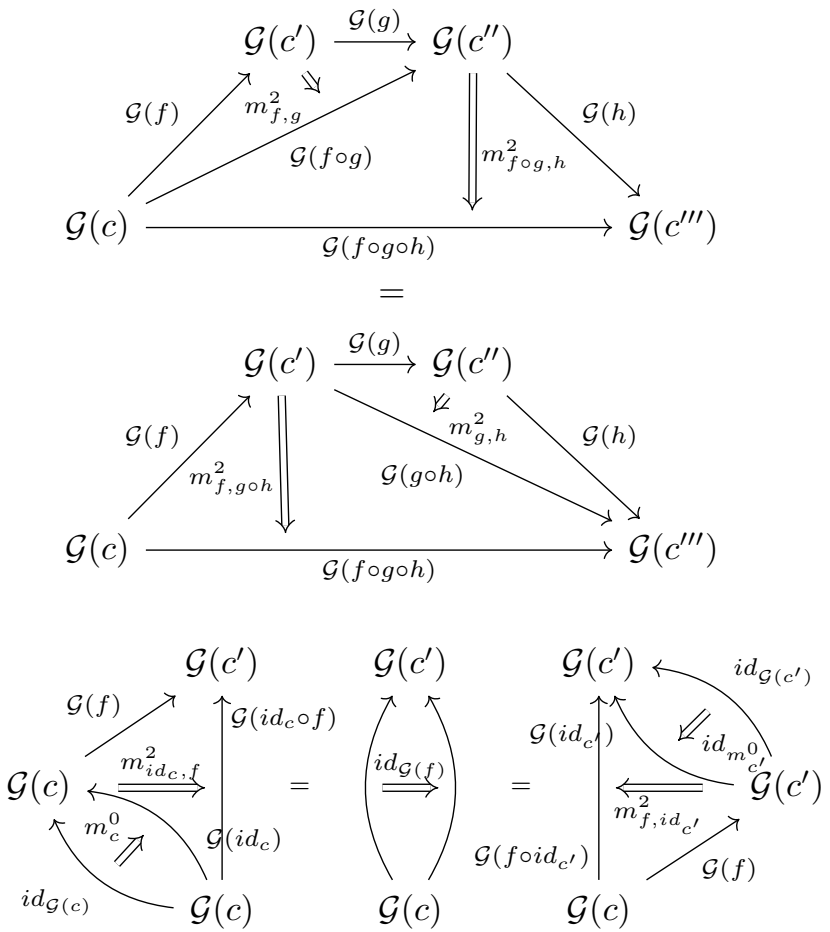

Returning to our construction of the the Grothendieck fibration for $\mathcal{F}$, we can keep the same definitions for 0 -cells, 1 -cells and 2-cells as in section III, that is :

- a 0 -cell consists of a pair $(b, m)$ of an object $b \in \mathcal{B}$ and a 1-cell $m: * \rightarrow \mathcal{F}(b)$ in $\mathcal{M} n d$,

- a 1-cell between $(b, m)$ and $\left(b^{\prime}, m^{\prime}\right)$ consists of a pair $(f, \varphi)$ where $f: b \rightarrow b^{\prime}$ is a 1-cell in $\mathcal{B}$ and $\varphi: m \rightarrow$ $\mathcal{F}(f) \circ m^{\prime}$ is a 2 -cell in $\mathcal{M} n d$

- a 2-cell between a parallel pair of 1-cells $(f, \varphi)$ and $(g, \psi)$ is a 2-cell $\alpha: f \rightarrow g$ in $\mathcal{B}$ such that the pasting of $\psi$ and $\mathcal{F}(\alpha)$ equals $\varphi$. 
We still need to equip this 2-globular structure with compositions and identities in order to get a 2-category and that is where the additional data provided with $\mathcal{F}$ comes into play. The identity on a 0 -cell $(b, m)$ is given by the pair $\left(i d_{b}, \iota_{(b, m)}\right)$ where $\iota_{b, m}$ is given by the following pasting diagram :

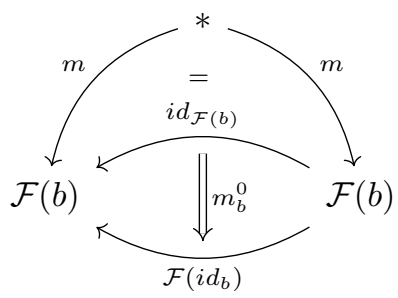

The composition of $(f, \varphi)$ and $(g, \psi)$ is the pair $(g \circ f, \xi)$ where $\xi$ is the following pasting diagram :

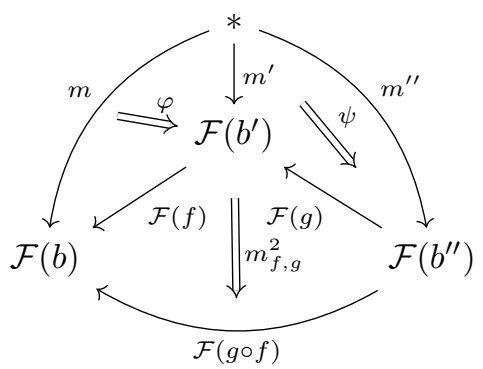

Vertical composition of 2-cells is given by its counterpart in $\mathcal{B}$ and horizontal composition is obtained from the naturality of $\mathrm{m}^{2}$. Associativity and unitality hold strictly since composition is associative and unital in $\mathcal{M} n d$ and the families of cells $m^{0}$ and $m^{2}$ are also associative and unital. 